\title{
Descripción organoléptica y anatómica de la madera de cinco especies forestales del sector Tabiazo, Esmeraldas,
} Ecuador @®@९

\author{
Organoleptic and anatomical description of the wood of five forest species \\ in the Tabiazo sector, Esmeraldas, Ecuador \\ Janella Carolina Chila Tenorio. ${ }^{1}$, Eduardo Patricio Salazar Castañeda. ${ }^{2}$, \& Víctor \\ Manuel Espinoza. ${ }^{3}$
}

Recibido: 04-05-2020 /Aceptado: 15-06-2020/ Publicado: 03-07-2020

\begin{abstract}
.
In Ecuador there are large areas of native forest and for some years these areas have been exploited indiscriminately, especially in the province of Esmeraldas. This research was based on organoleptically and anatomically describing the wood of five forest species, coming from the Tabiazo parish, Esmeraldas canton, province of the same name; to generate technical information on the macro and microscopic characteristics of different forest species. To dendrologically identify the five forest species under study, the collection of botanical specimens from the herbarium of the Chimborazo Polytechnic was used. To describe the anatomical characteristics of the five species, cuts were made according to the standards COPANT (1972). The same ones that were exposed to a softening process in a humid autoclave at $121^{\circ} \mathrm{c}$ and $1.1 \mathrm{~atm}$, then in the microtome histological cuts were made to obtain 0.3 micron sheets, they were placed in staining based on a combination of Safranina and Astrablue, while the senses were used to describe the organoleptic characteristics. Motic images 2.00 were used to determine which stain allows the anatomical characteristics to be observed in a better way. Finally, it is concluded that in dendrological identification none of the species belongs to the same family. The organoleptic and anatomical characteristics are different and all the species
\end{abstract}

\footnotetext{
${ }^{1}$ Ingeniera Forestal, Investigador independiente, Ecuador, janellachila93@ gmail.com, ORCID: 00000002-6239-6958

${ }^{2}$ Escuela Superior Politécnica de Chimborazo, eduardo.salazar@espoch.edu.ec, ORCID: 00000001-7737-5415

${ }^{3}$ Escuela Superior Politécnica de Chimborazo, victor.espinoza@espoch.edu.ec, ORCID: 0000-0001-60582274
} 
presented solitary and multiple pores of two and three and one of them presented pores of four. The best staining was combined since structures with better quality are observed.

Keywords: Wood, Organoleptic characteristics, Forest species, Histological sections.

\section{Resumen.}

En Ecuador existe grandes áreas de bosque nativo y desde hace algunos años estas áreas de han sido explotadas indiscriminadamente especialmente en la provincia de Esmeraldas. Esta investigación se basó en describir organoléptica y anatómicamente la madera de cinco especies forestales, procedentes de la parroquia Tabiazo, cantón Esmeraldas, provincia del mismo nombre; para generar información técnica sobre las características macro y microscópicas de diferentes especies forestales. Para identificar dendrologicamente las cinco especies forestales en estudio se utilizó la colección de especímenes botánicos del herbario de la Politécnica de Chimborazo. Para describir las características anatómicas de las cinco especies se realizó cortes de acuerdo con las normas COPANT, (1972). Las mismas que se expusieron a un proceso de ablandamiento en una autoclave húmeda a $121^{\circ} \mathrm{C}$ y $1.1 \mathrm{~atm}$, luego en el micrótomo se realizaron cortes histológicos para obtener láminas de 0.3 micras, las mismas se procedió a colocarlas en tinción a base de una combinación de Safranina y Astrablue, mientras que para describir las características organolépticas se utilizó los sentidos. Para determinar que tinción permite observar las características anatómicas de mejor forma se utilizó Motic ímages 2.00. Finalmente se concluye que en la identificación dendrológica ninguna de las especies pertenece a la misma familia. Las características organolépticas y anatómicas son diferentes, de todos los ejemplares estudiados se encontró que tenían poros solitarios y estos múltiples de dos y en otros casos de tres. una de ellas presento poros de cuatro. La mejor tinción fue combinada ya que se observan estructuras con mejor calidad.

Palabras clave: Madera, Características organolépticas, Especies forestales, Cortes histológicos.

\section{Introducción.}

El En el Ecuador existen grandes áreas de bosque nativo ubicadas especialmente en la Región Amazónica, en las estribaciones externas de las dos cordilleras de la región andina y en zonas secas y húmedas de la Costa. Estos bosques son los suministradores de productos forestales como: maderas, leña, frutos, resinas, cortezas, bejucos, carnes, pieles y más elementos de la flora y fauna silvestres. Así mismo, proporciona el espacio vital en el que se asientan numerosas poblaciones de indígenas y colonos para quienes estos recursos constituyen importantes fuentes de subsistencia y de ingresos. De los esfuerzos que se realicen para la conservación de los bosques dependerá en gran medida el futuro del ambiente y el uso sostenible de los recursos naturales del país (Zúñiga, 1997).

El uso y comercio de la madera y sus derivados son actividades que han estado presentes en la economía del país. El Ecuador ha sido autosuficiente en productos madereros, 
gracias a una base forestal que originalmente cubría el $80 \%$ del territorio nacional. Esta situación actualmente se está revirtiendo debido a conocidos problemas de sobreexplotación y tala irracional de bosques nativos, frente a una incipiente forestación (Zuñiga, 1997).

La falta de recursos económicos en nuestro país ha llevado a la población a buscar otras fuentes de ingreso, y una de ellas ha sido la explotación forestal de manera inadecuada. (MAE. 2020). Por este motivo es importante realizar estudios anatómicos y organolépticos de la madera para proporcionar información correcta y específica de las especies forestales, esta investigación tiene como propósito generar información científica sobre las características macro y microscópicas de diferentes especies forestales y contribuir a realizar un adecuado aprovechamiento de este recurso forestal. De allí que el objetivo del presente estudio es describir organoléptica y anatómicamente la madera de cinco especies forestales, del sector Tabiazo, Esmeraldas, Ecuador.

El recurso forestal desde siglos pasados se ha sido utilizado como fuentes primarias de material para la construcción de viviendas, muebles y herramientas. En nuestros días el requerimiento de este recurso se encuentra en aumento. Especialmente se debe destacar las especies que se las puede encontrar en los bosques tropicales húmedo de la región ecuatorial. Las mismas que son aprovechadas sin conocer las propiedades anatómicas, físicas y mecánicas. (Agila, Burneo, Narvaes, \& Pucha-Cofrep, 2018).

El conocimiento de las características anatómicas de las maderas, así como el de sus propiedades químicas y mecánicas, aparte del beneficio científico que lo rodea son de importancia industrial para un país. Cada familia, género o especie de árbol produce madera que tienen propiedades y usos apropiados, que solamente pueden ser determinados en forma eficiente por medio de la investigación (Acosta, 1967).

\section{Anatomía de la madera.}

Para realizar los estudios anatómicos de la madera Chavesta (2006), indica que necesario realizar cortes en diferentes planos. En el árbol y células de la xilema se pueden definir tres cortes o planos. Para el corte transversal Hoadley, B (1990), menciona que en este corte se puede ver los anillos de crecimiento, tamaño de los radios, también se puede apreciar la porosidad, parénquima, hasta tejidos conductores como el floema y también la diferente coloración que se observa en la albura y el duramen. En referencia a los cortes radial y tangencial Gimenez et. al., (2005), indica la incisión va en relación paralela a los canales radiales de la madera, Además de la apariencia, también el comportamiento físico- mecánico de la madera difiere en cada uno de estos sentidos, fenómeno conocido como anisotropía. Por presentar esta particularidad, la madera es un material anisotrópico.

\section{Propiedades organolépticas de la madera.}

Las propiedades organolépticas corresponden a las que se perciben por los sentidos el color para (Tknika, 2006), el color de la madera depende del color de las paredes de sus 
células y del contenido intercelular y es una característica de cada especie. En la madera pueden ser distintos el color de la albura y el del duramen. Para Giménez, A., Moglia, J., \& Bravo, S. (2014) Mientras el olor se genera por los aceites y alcoholes que se evaporan fácilmente, pero esto es variable por los distintos niveles de percepción de los técnicos que estén realizando las pruebas por lo que es recomendable reportar solo cuando son fácilmente apreciables, además se debe tener en cuenta que estas características se pierden con el tiempo. El sabor según (Aguilar \& Guzowski, 2011). no es una característica muy habitual en las maderas, pero se encuentra relacionado con el aroma, en los dos casos son producidos por los aceites esencias de las especies.

El brillo o lustre (Chavesta, 2006), indica que es la capacidad de emitir el brillo producido por la luz. El lustre está relacionado por la variedad de células expuesta en relación a los rayos luminosos con cierta inclinación. Mientras que la textura (Cloquell et al., 2005), indica que se refiere al tamaño, cantidad de células distribuidas en la superficie de la muestra. Las maderas de poro pequeño, radios finos y escaso parénquima se consideran como de textura fina. Poros grandes, gran cantidad de parénquima y radios anchos es de textura gruesa. Y para el grano (Moglia et al., 2014), indica que es la trayectoria en la misma dirección la madera sea en cortes tangenciales o radiales.

La safranina es un colorante catiónico que aporta color rojo a las estructuras histológicas. Es muy usada en histología vegetal donde tiñe de rojo las células lignificadas como, los núcleos y la lignina de las paredes celulares secundarias. La safranina colorante bilógico de contraste para producir un color violeta intenso utilizado sobre otras tinciones (Aguirre, 2012). Y el Astra blue colorante que se liga de mejor manera con la sacarosa en los vegetales lo produce un color azul intenso así mismo es utilizado en muestra de células sanguíneas (Feijoo, Pucha, \& Ramon, 2018).

\section{Metodología.}

\section{Características organolépticas.}

Para la obtención de las muestras de las maderas se consideró obtener probetas con las dimensiones de $15 \mathrm{~cm}$. x 9cm. $2 \mathrm{~cm}$, de acuerdo con las normas COPANT, (1972). Las características organolépticas se determinaron utilizando los sentidos: olfato, tacto, gusto y vista. El color se observó utilizando la tabla de Munsell. Según el criterio Hoadley, B. (1990). Para observar las características organolépticas en las muestras se utilizan los sentidos es decir son apreciables a simple vista, estas características son: color, olor, brillo y veteado. El color fue analizado la paleta de colores de Munsell. El olor se evaluó en aromático, astringente, fragante y dulce. En cuanto al sabor se evaluó en picante, agrio, dulce, desagradable, agradable y amargo, cabe señalar que en algunas especies el olor y sabor suele estar ausentes. Para el lustre se evaluó en alto medio y bajo, el veteado se catalogó en jaspeados satinados o bandas paralelas y arcos superpuestos (Lema, E. 2018). 


\section{Características anatómicas.}

Se obtuvieron probetas de madera con las siguientes dimensiones $2 \mathrm{~cm}$ x $2 \mathrm{~cm}$ x $2 \mathrm{~cm}$, de acuerdo a las normas COPANT (1972), de las cinco especies (laurel, teca, amarillo, caoba, guachapeli) con sus cuatro repeticiones. se pesaron los cubos separados por corte y se colocó agua destilada en la autoclave a un nivel conocido y esperar que caliente. Los cubos se colocaron dentro de los frascos de vidrio y se añadió $200 \mathrm{ml}$ de agua destilada, posteriormente se taparon los frascos con papel aluminio, se etiquetaron y luego se los colocó en la autoclave húmeda a 16.16 PSI y a temperatura constante de 121 grados centígrados por ciclos de 20 minutos.

Luego se realizaron los cortes (tangencial, radial y longitudinal) en el micrótomo rotatorio con un grosor de 0,3 micras cada muestra, según las normas IAWA. (2016). Hubieron especies a las que necesitó llevar a un proceso de congelamiento para poder realizar el corte, a la Teca (Tectona grandis) por veinte y cuatro horas después del octavo ciclo (para que se diera un choque térmico) para poder realizar el corte deseado y al Laurel (Cordia alliodora) por veinte y cuatro horas después de su primero y único ciclo, debido a que esta especie no se encontraba muy lignificada y al realizar los cortes en el micrótomo sin congelar las láminas no cumplían los estándares; en el caso de la especie Guachapeli (Leucaena trichodes) se la mantuvo en congelación por diez minutos.

Las tinciones se colocaron en cajas Petri y luego se ubicaron las láminas de madera obtenidas de los diferentes cortes histológicos hasta que se cubrieron totalmente con la tinción, se dejó reposar las muestras 8 minutos en la tinción (safranina + astrablue usando una proporción de 1:1, safranina y astrablue), también se colocó agua destilada y alcohol al $70 \%$ en diferentes cajas Petri, cada una etiquetada respectivamente, una vez retiradas las muestras de las tinciones se las colocó en agua destilada para enjuagar las muestras. Con cada uno de los lentes del microscopio Quimis Motic, se observó las estructuras anatómicas, la cantidad en la que se encuentran los poros, el tipo de parénquima, los radios, punteaduras, tílides, células envolventes, y demás estructuras.

Con el software Motic Images Plus 2.0. Se calibró en relación micrómetro/pixel y utilizando una imagen capturada de buena calidad, se elaboró una base de datos en Excel por especie (total de poros, poros solitarios y múltiples de 2, 3 y 4). Luego mediante el infostat para el análisis comparativo entre especies mediante bloques completamente al azar. Además, una prueba de Normalidad de Shapiro-Wilks que sirvió para demostrar si los datos siguen una distribución normal para luego hacer el análisis de varianza.

\section{Características físicas.}

Para la determinación del pH se obtuvo aserrín fresco de cada especie, luego se pesó en una balanza digital 20 gramos de aserrín y se ubicó en diferentes vasos cada especie, Seguidamente se colocó $50 \mathrm{ml}$ de agua destilada agitando hasta formar una solución homogénea, Posteriormente se tomó la medición con un $\mathrm{pH}$ metro, donde se obtuvo la medida exacta del valor de $\mathrm{pH}$ de la solución de cada especie. 


\section{Resultados.}

Los resultados en referencia a las características organolépticas de las cinco especies estudiadas lo podemos ver detalladamente en la tabla 1. las mismas que se obtuvieron de acuerdo con la percepción de nuestros sentidos.

En Senna macroplylla se determinó que es una Especie de fuste recto, corteza agrietada de color café oscuro. En la paleta de colores la albura marco correspondiente a $5 \mathrm{Y}$ 8/4 y 2,5 Y 7/6 para el duramen, el brillo con una intensidad media, grano de la fibra ondulado, textura gruesa, la veta de la madera jaspeada, olor y sabor no distintivo en algunas especies. Para Cordia alliodora Esta especie presenta fuste cilíndrico, su corteza de color marrón y manchas blancas, no fisuradas; En la paleta de colores el color marron para la albura 10 YR 8/1 y marrón amarillento 10YR 7/3 para el duramen, brillo intenso, textura media y grano recto, olor agradable. y sabor ausente. Mientras que en Centrolobiu ochoxylum es una especie que presenta fuste recto, su corteza es de marrón claro y partida, la paleta de colores marca la albura $5 \mathrm{Y} 8 / 3$ de tono amarrillo y el duramen $2.5 \mathrm{y}$ $8 / 8$ de tono amarrillo y referente al brillo medio, grano entrecruzado, textura gruesa, veteado jaspeado, olor agradable no distintivo y sabor ausente. En Tectona grandis Especie que posee una corteza escamosa de color castaño y blanquecina; la paleta de colores la albura marca 10YR 8/4 y el duramen 10YR 7/4 tono castaño, su brillo medio, grano recto / inclinado, textura media, veteado jaspeado con bandas paralelas, olor y sabor ausente. En Leucaena trichodes. Esta especie presenta una corteza fisurada de color café grisaceo, según la paleta de Munsell el color de la albura 5Y 8/6 con un tono amarrillo. 7.5YR 6/6 para el duramen con un tono amarrillo - rojo 7.5 7YR 6/6 brillo medio, grano entrecruzado, textura media, veteado jaspeado, olor agradable no distintivo y sabor cercano a frijoles.

Tabla 1. Características organolépticas de las cinco especies forestales estudiadas.

\begin{tabular}{|c|c|c|c|c|c|}
\hline $\begin{array}{l}\text { Características } \\
\text { Organolépticas }\end{array}$ & $\begin{array}{l}\text { s Senna } \\
\text { smacroplylla }\end{array}$ & $\begin{array}{c}\text { Cordia } \\
\text { alliodora }\end{array}$ & $\begin{array}{c}\text { Centrolobium } \\
\text { ochoxylum }\end{array}$ & $\begin{array}{l}\text { Tectona } \\
\text { grandis }\end{array}$ & $\begin{array}{l}\text { Leucaena } \\
\text { trichodes }\end{array}$ \\
\hline Corteza & $\begin{array}{l}\text { Agrietada, } \\
\text { café oscuro. }\end{array}$ & $\begin{array}{l}\text { Manchas } \\
\text { blancas, } \\
\text { marrón }\end{array}$ & $\begin{array}{c}\text { Fisurada, } \\
\text { marrón claro. }\end{array}$ & $\begin{array}{c}\text { Escamosa, } \\
\text { castaño y } \\
\text { blanquecina. }\end{array}$ & $\begin{array}{l}\text { Fisurada, } \\
\text { grisáceo. }\end{array}$ \\
\hline Albura & $\begin{array}{l}\text { Amarillo } \\
\text { pálido }\end{array}$ & $\begin{array}{c}\text { Marrón } \\
\text { pálido }\end{array}$ & Amarillo & $\begin{array}{c}\text { Marrón muy } \\
\text { pálido }\end{array}$ & $\begin{array}{c}\text { Marrón } \\
\text { amarillento }\end{array}$ \\
\hline Duramen & Amarillo & $\begin{array}{c}\text { Marrón } \\
\text { amarillento }\end{array}$ & $\begin{array}{l}\text { Amarillo } \\
\text { intenso }\end{array}$ & Castaño & $\begin{array}{c}\text { amarillo } \\
\text { rojizo }\end{array}$ \\
\hline Veteado & Jaspeado & $\begin{array}{c}\text { En arcos } \\
\text { superpuestos. }\end{array}$ & Jaspeado & $\begin{array}{c}\text { Jaspeado en } \\
\text { bandas } \\
\text { paralelas. }\end{array}$ & Jaspeado \\
\hline Textura & Gruesa & Media & Gruesa & Media & Media \\
\hline Grano & Ondulado & Recto & Entrecruzado & $\begin{array}{l}\text { De recto a } \\
\text { inclinado }\end{array}$ & Entrecruzado \\
\hline Lustre & $\begin{array}{l}\text { De medio / } \\
\text { alto }\end{array}$ & Medio & Medio & Medio & Medio \\
\hline
\end{tabular}




\begin{tabular}{cccccc}
\hline Sabor & $\begin{array}{c}\text { Ausente } \\
\text { Agradable } \\
\text { no }\end{array}$ & Ausente & Ausente & Ausente & A frijoles \\
distintivo. & Ausente & $\begin{array}{c}\text { Agradable no } \\
\text { distintivo. }\end{array}$ & Ausente & $\begin{array}{c}\text { Agradable } \\
\text { no distintivo. }\end{array}$ \\
\hline
\end{tabular}

Fuente: Elaboración propia.

\section{Senna macroplylla (Caoba)}

En el corte Trasversal figura 1, podemos apreciar en el literal a. Con tinción astrablue y observado con el lente óptico de 10x, poros múltiplos de dos con parénquima paratraqueal aliforme de ala ancha y corta, radios finos. En b. Con la tinción Safranina, observado con el lente 10x, poros múltiplo de dos y tres. En c. Observado con el lente 4x y tinción combinada se tiene disposición de los poros sin patrón definido, poros solitarios con parénquima paratraqueal vasicéntrico y poros múltiplos de 2 , parénquima paratraqueal vasicéntrico confluente.

Figura 1. Corte Transversal Senna macroplylla (Caoba).

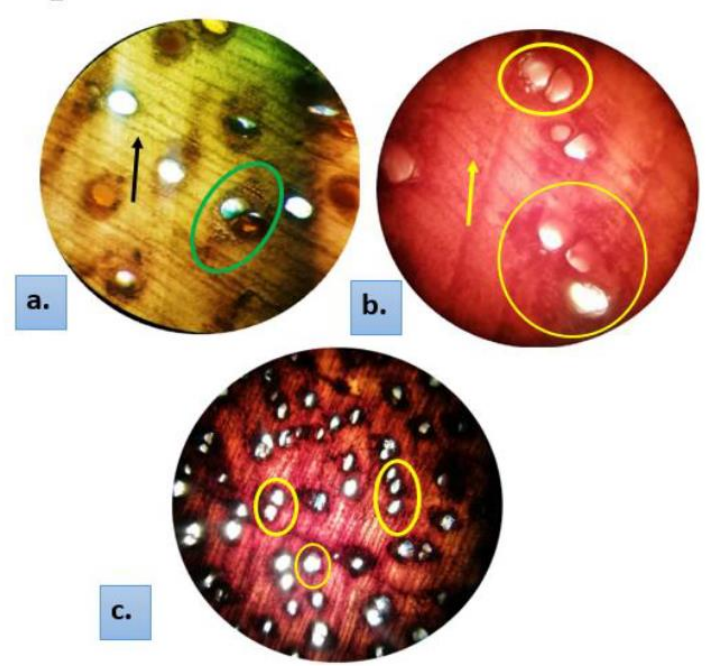

Fuente: Elaboración propia.

En el corte longitudinal Observado con el lente de 4x, con tinción de safranina + astrablue, se observa radios uniseriados con presencia de células envolventes de los radios y se observa el miembro del vaso con punteaduras. Observado con el lente 10x, con tinción safranina + astrablue, radios uniseriados heterogéneos y miembros del vaso con puntuaduras.

En el corte longitudinal Observado con el lente óptico 10x, con la tinción safranina + astrablue, se encuentra célula con radios homogéneos. Observado con el lente óptico 4x, con la tinción safranina, se puede apreciar la fibra de los radios no septadas y septadas. 


\section{Cordia alliodora (Laurel)}

Corte transversal en a observado con el lente óptico 10x, y tinción safranina + astrablue se tiene poro solitario con parequima paratraqueal vasicentrico, radios gruesos, en $b$. Observado con el lente óptico 4x, y tinción astrablue + safranina, poros múltiplos de dos es su mayoría, seguido de poros solitarios con parénquima paratraqueal vasicentrico. Mientras que observado con el lente óptico $4 \mathrm{x}$, y tinte astrablue, poros solitatios $\mathrm{y}$ múltiplos de dos, con porosidad difusa uniforme. Como se observa en la figura 2.

Figura 2. Corte Transversal Senna macroplylla (Caoba).

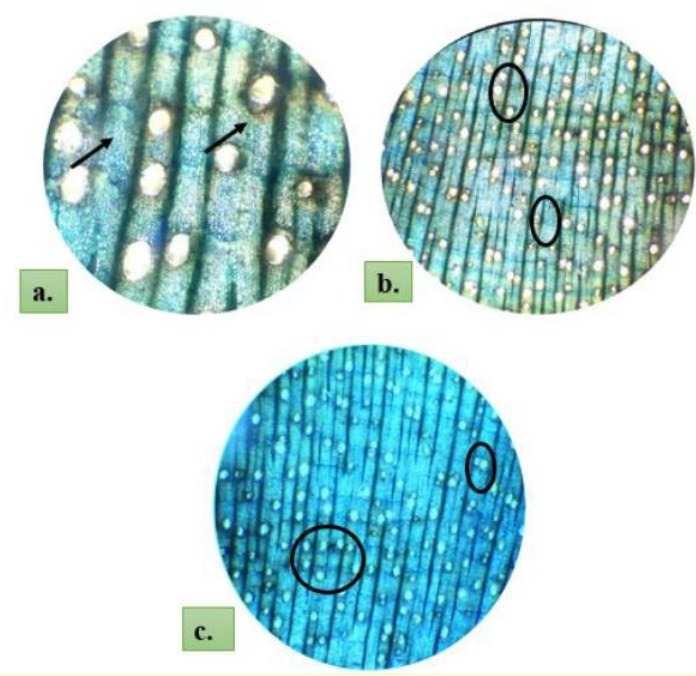

Fuente: Elaboración propia.

En el corte tangencial observado con el lente $4 \mathrm{x}$ radios biseriados y multiseriados, con la tinción safranina + astrablue. Con el lente óptico 10x, y tinte safranina + astrablue radios multiseriados con células envolventes, con el lente óptico 40x con la tinción astrablue se tiene radios multeseriados con células envolventes y radios agregados. En el corte longitudinal observado con el lente óptico 10x con tinción safranina + astrablue. Se obtiene fibras septadas. Observado en el microscopio de 40x presencia de punteaduras en el vaso.

Según Richter \& Dallwitz (2000), manifiestan de las características anatómicas de Cordia alliodora, en su estudio realizado presento porosidad semicircular, parénquima axial en bandas, parénquima paratraqueal vasicéntrico confluente y radios multiseriados. El estudio tiene relación con los resultados de la presente investigación a excepción del parénquima axial en bandas, en los resultados obtenidos se adquirió un parénquima paratraqueal unilateral. 


\section{Centrolobium ochoxylum (Amarillo)}

Observado en corte trasversal en la imagen a. con tinción safranina y el lente óptico 4x, se mira poros múltiplos de tres en su gran mayoría, seguidos de poros solitarios y muy escasos poros múltiplos de dos de tamaño medio. En b. Observado con el lente óptico 40x y tinción safranina, radios de tamaño medio y poros múltiplos de tres en su gran mayoría. En c. observado con el lente 10x y tinción safranina + astrablue, poros con parénquima paratraqueal vasicéntrico confluente, poro solitario con presencia de tílides. Como muestra la figura 3.

Figura 3. Corte Transversal Senna macroplylla (Caoba).

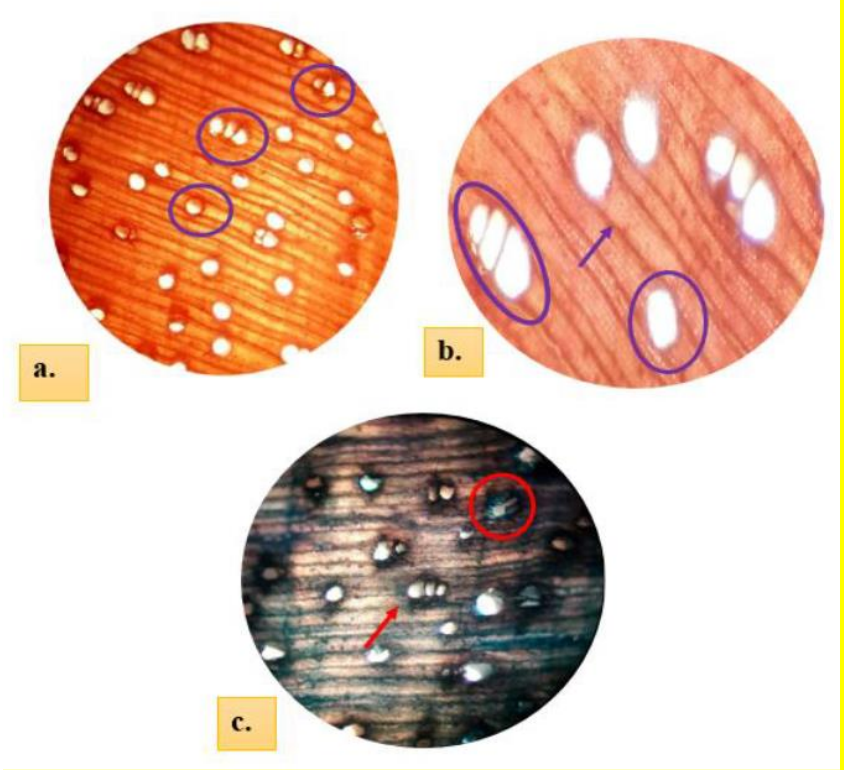

Fuente: Elaboración propia.

En el corte tangencial observado con el lente óptico 4x, con la tinción safranina+ astrablue se puede distinguir células parenquimáticas. Observado con el lente óptico 10x, células parenquimáticas con fibras septadas heterogéneas. y con el lente de 10x, células parenquimáticas con fibras septadas, y radios homogéneos.

En el corte longitudinal observado con el lente óptico de 4x y tinción safranina+ astrablue, radios homogéneos compuestos con células procumbentes. Mientras que con el lente 10x con la tinción astrablue, se observa radios homogéneos compuestos y puntiaduras ornadas. Y con el lente óptico 40x se distingue de mejor manera los radios homogéneos.

\section{Tectona grandis (Teca)}

De acuerdo con la figura 4, en a. mirando con el lente óptico de 4x y la tinción astrablue, se puede distinguir poros múltiplo de dos en su mayoría seguido de poros solitarios, con presencia de tílides. En b. con el lente 10x, con tinción safranina + astrablue, una 
porosidad circular donde los poros de mayor tamaño están en orientación a los anillos de crecimiento. En c. Observado con el lente 40x y tinción safranina + astrablue, poros solitarios con parénquima paratraqueal vasicentrico y múltiplo de dos con parénquima paratraqueal vasicentrico confluente.

Figura 4. Corte transversal de Tectona grandis (Teca)

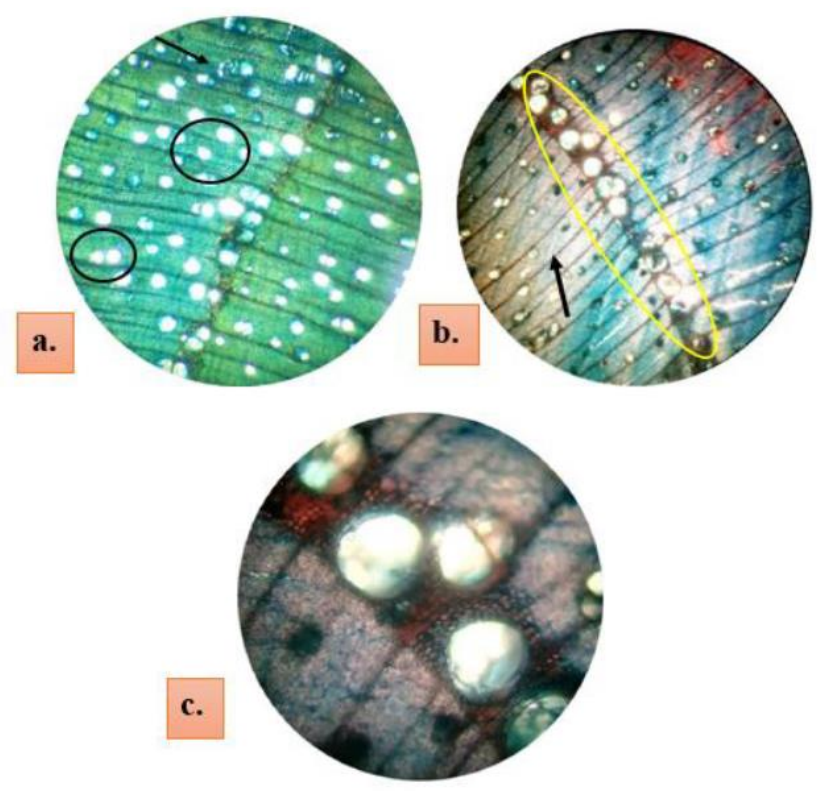

Fuente: Elaboración propia.

En el corte tangencial con el lente 4x y la tinción combinada se pudo observar radios multiseriados, presencia de puntiaduras. Con el lente óptico de 10x y la tinción combinada se puede observar radios multiseriados y la intersección de las fibras con las células procumbentes.

En el corte longitudinal observado con el lente 10x y la tinción combinada, se observa elementos de vasos con muy pocas puntiaduras y radios heterogéneos. Con el lente óptico 4x se observan radios multiseriados. Con el lente óptico de 10x y la tinción astrablue se logra distinguir elementos del vaso con muy poca cantidad de puntiaduras.

Según Bhat, K., Priya, P., \& Rugmini, P. (2001) en investigaciones realizadas en características organolépticas, argumenta que en Tectona grandis en la albura presenta un color de blanco a amarillo pálido, mientras que el duramen de blanquecino a castaño, en ello indica que la madera proviene de árboles de mayor edad. La investigación realizada comparte características similares con nuestro estudio de características organolépticas

\section{Leucaena trichodes (Guachapali)}

En la figura 5 con el corte transversal observado con el lente óptico 4x y la tinción combinada, poros múltiplos de dos en su mayoría, seguido de poros múltiplos de tres con parenquima patraqueal vasicentrico confluente y en menor cantidad poros solitarios con 
parénquima paratraqueal vasicentrico. En b. Observado con el lente óptico de $10 \mathrm{x}$, radios medianamente finos y un poro obstruido. Y en c. Observado con el lente 10x, radios medianos con fibras de paredes medianas.

Figura 5. Corte transversal de Leucaena trichodes (Guachapeli)

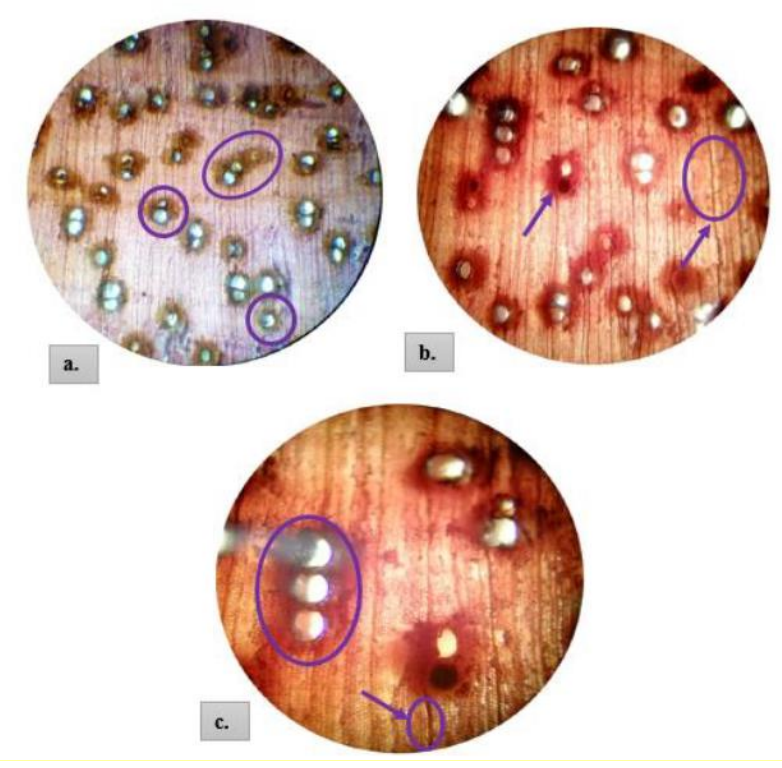

Fuente: Elaboración propia.

En el corte tangencial observado con el lente 4x y la tinción combinada, radios heterogéneos multiseriados. Observado con la tinción astrablue y el lente óptico 4x, radios multiseriados. y Observado con el lente 10x y la tinción combinada radios multiseriados estratificados.

En el corte longitudinal con el lente óptico 4x y la tinción combinada, se puede observar radios heterocelulares. Observado con el lente 4x y la tinción astrablue, presencia del vaso con escasas punteaduras.

Mediante el programa InfoStat se seleccionaron los datos de los poros solitarios y múltiples, posteriormente se efectúo el análisis de varianza y se observó que existen diferencias significativas en los datos de cada especie. Luego se realizó el análisis correspondiente a la normalidad de Shapiro obteniendo los resultados con una distribución normal, es decir p>0,05.

\section{Conclusiones:}

- Las especies presentan brillo de medio a alto. Cuatro de las cinco especies presentan la característica de sabor ausente solo Leucaena trichodes tiene sabor a frijoles. En la característica referente al olor es agradable no distintivo para Senna macrophylla, Leucaena trichodes y Centrolobium ochoxylum y ausente en Tectona grandis y Cordia alliodora. 
- Las características de los poros en 4 especie en el corte transversal se encuentra poros solitarios múltiplos de tres y en Centrolobium ochoxylum (Amarillo) se observa poros múltiplos de cuatro. De acuerdo al tamaño de los poros Senna macroplylla (Caoba), Leucaena trichodes (guachapeli) y Centrolobium ochoxylum (Amarillo) presenta poros de tamaño mediano, Cordia alliodora (laurel) poros pequeños y Tectona grandis (teca) muestra que su poro tiene tamaño variado donde los poros de mayor tamaño están en orientación a los anillos de crecimiento y los que se encuentran difusos son de tamaño mediano.

- El parénquima de cuatro especies es similar en paratraqueal vasicéntrico, excepto Senna macrophylla que posee parénquima paratraqueal aliforme de ala ancha y corta. Las cinco especies presentan radios no estratificados.

- Las tres tinciones son efectivas; pero entre ellas que resulta mejor su uso es la combinación de safranina más atrablue debido a que reacciona de mejor manera en las especies forestales y en sus diferentes cortes.

\section{Referencias bibliográficas:}

Acosta, I. (1967). Descripción anatómica. propiedades físicas. Costa Rica: Instituto Interamericano de Ciencias Agrícolas de La OEA. p. 201.

Agila, S., Burneo, A., Narvaes, L., \& Pucha-Cofrep, D. (2018). Propiedades físicas y características anatómicas de la madera de tres especies de Guayacán al sur de Ecuador. Bosques Latitud Cero, 8(2), 1-15

Bhat, K., Priya, P., \& Rugmini, P. (2001). Characterization of juvenile wood in teak. Wood Science and Technology 34(6):517-532.

Cloquell, V. A., Contreras, W., \& Owen, M. (2005). La madera y los productos forestales en sistemas estructurales. Madrid, España.

Comisión Panamericana de Normas Técnicas. (1972). Normas técnicas, 458 maderas de selección y colección de muestras. New York: COPANT

Chavesta, C. (2006). Separata de capacitación sobre identificación de madera. Perú. pp. 8-13.

Giménez, A., Moglia, J., \& Bravo, S. (2014). Tomo II macroscopia de la Madera. Santiago del Estero, Argentina.

Hoadley, B. (1990). Identifying Wood. The Taunton Press, Inc. Newtown. U.S.A. Recuperado el 17 de enero de 2019, de https://www.researchgate.net/publication/44368335_Anatomia_de_la_madera_ Willia ms_Jose_Leon_Hernandez_Narcisana_Espinoza_de_Pernia

IAWA. (2016). List of microscopic bark features, International Association of Wood Anatomists Journal, 37(4), 517-615.

Lema, Estrella. (2018). Caracterización anatómica; Trophis caucana; Rollinia dolichopetala; Maclura tinctoria; Clarisia racemosa; parque botánico regional Sucúa; Morona Santiago. (tesis de pregrado). Escuela Superior Politécnica de Chimborazo, Ecuador. 
MAE. (2013). Sistema Nacional de Control Forestal Recuperado el 27 de junio de 2020 de https://www.ambiente.gob.ec/wpcontent/uploads/downloads/2015/07/CONTROL-FORESTAL.pdf

Richter, G., \& Dallwitz, M. (2000) Onwards. Commercial timbers: descriptions, illustrations, identification, and information retrieval. In English, French, German, Portuguese, and Spanish. Version: 9th April 2019.

Zuñiga, T. (1997). Principales Estadísticas Forestales del Ecuador. Dirección General de Planificación. Quito, Ecuador. 


\section{PARA CITAR EL ARTÍCULO INDEXADO.}

Chila Tenorio, J. C., Salazar Castañeda, E. P., \& Espinoza, V. M. (2020). Descripción organoléptica y anatómica de la madera de cinco especies forestales del sector Tabiazo, Esmeraldas, Ecuador. ConcienciaDigital, 3(3), 164-177.

https://doi.org/10.33262/concienciadigital.v3i3.1288

\section{Liencia}

El artículo que se publica es de exclusiva responsabilidad de los autores y no necesariamente reflejan el pensamiento de la Revista Conciencia Digital.

El artículo queda en propiedad de la revista y, por tanto, su publicación parcial y/o total en otro medio tiene que ser autorizado por el director de la Revista Conciencia Digital.

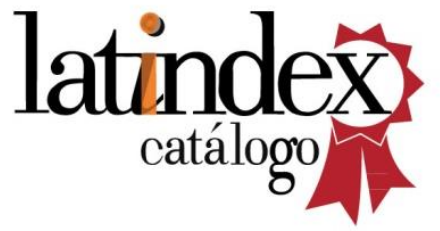

